\title{
Rheumatoid nodules of the vocal folds
}

\author{
JOHN WEBB* AND W. H. PAYNE $\dagger$ \\ The Sutton Rheumatism Research Laboratory and the Department of Pathology, Royal North Shore \\ Hospital, Sydney, Australia
}

Rheumatoid involvement of the larynx occurs frequently and causes a wide variety of symptoms. These include hoarseness, dyspnoea, stridor, dysphagia, a sensation of fullness or of a foreign body in the throat, and throat pain which may radiate to the ears and occur on speaking. Such symptoms are usually due to rheumatoid arthritis involving the cricoarytenoid joints (Lofgren and Montgomery, 1962). Laryngeal polymyositis and ischaemic recurrent laryngeal nerve paresis have also been described in rheumatoid arthritis (Wolman, Darke, and Young, 1965). Though nodules and polyps have been noted in and around the larynx in such cases, rheumatoid nodules involving the vocal folds do not appear to have been specifically described in the past. Their occurrence in a patient with classical rheumatoid arthritis is reported below.

\section{Case report}

A woman now aged 48 years had developed a chronic productive cough in 1961 at the age of 38 . She had previously been healthy and without respiratory symptoms. Bronchography subsequently demonstrated bilateral bronchiectasis which has since been managed with regular postural drainage and long-term antibiotics. In 1965 she developed seropositive, nodular, erosive rheumatoid polyarthritis. Therapy was begun with regular high-dose salicylates and indomethacin, and initially included a course of chrysotherapy. Continued activity of the arthritis led to the introduction of prednisone in 1968, and this has been continued at a dose of $10 \mathrm{mg}$. daily. In 1967 hypothyroidism became manifest and replacement therapy with 1-thyroxine has also been continued.

In February, 1970, there was a severe exacerbation of the rheumatoid arthritis associated with the appearance of digital arteritic lesions of the nailfolds, pitting rheumatoid oedema of the limbs, and a sparse maculopapular skin rash.

\section{Laboratory investigations}

Haemoglobin 11.6 g. $/ 100 \mathrm{ml}$; total leucocyte count 9,700 per c.mm. with normal differential count; erythrocyte sedimentation rate $90 \mathrm{~mm}$./1 st hr; platelet count greater than $1 \times 10^{6}$ per c.mm.; marked Rouleaux formation.
The Waaler-Rose titre was $1 / 10,000$, and serum protein electrophoresis revealed marked diffuse hypergammaglobulinaemia.

\section{Treatment}

The immunosuppressive drug cyclophosphamide was given and there was some slow improvement, which continued until November, 1970, when she was hospitalized for metacarpophalangeal synovectomies.

\section{Laryngeal involvement}

In the post-operative period, a complaint of persistent hoarseness of some 8 months' duration was investigated by Mr. J. Dowe, Honorary E.N.T. Surgeon. At laryngoscopy the vocal folds moved normally. A nodule was present in the substance of each vocal fold at about its midpoint, but not opposing each other; these were unlike any previously seen (Fig. 1). These nodules were removed by curettage (Fig. 2); the hoarseness improved and the vocal folds now appear normal

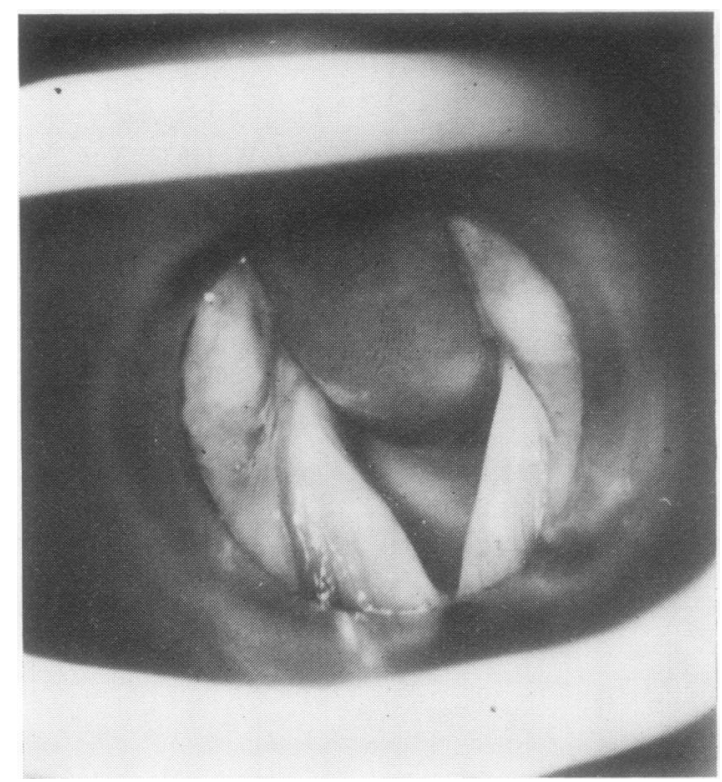

FIG. 1 Appearance on direct laryngoscopy. There is a nodular swelling within the substance of each vocal fold at about their mid-points 


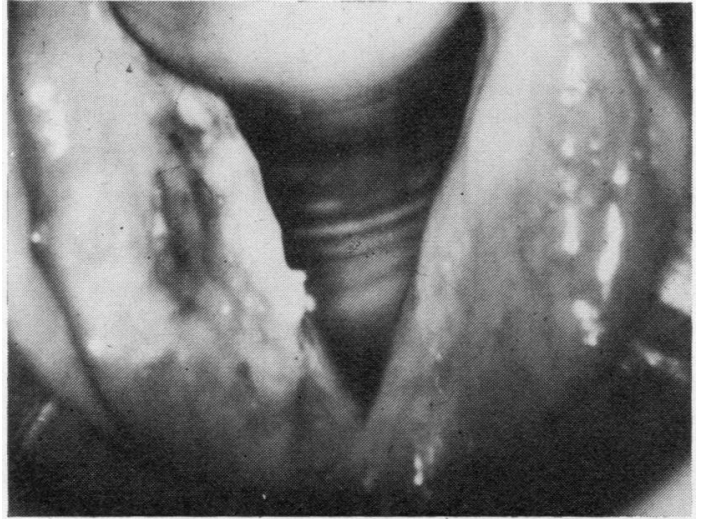

I FIG. 2 Appearance on direct laryngoscopy. The nodule in the right vocal fold has been curetted out. The nodular swelling within the substance of the left vocal fold is more obvious

\section{Progress}

In February, 1971, the patient developed livido reticularis and other skin lesions of necrotizing arteritis (confirmed on biopsy) complicating a further severe exacerbation of rheumatoid disease, for which she is now receiving highdosage steroids.

\section{Pathology (Figs 3 and 4)}

The biopsies from the vocal folds were embedded in paraffin wax and $6 \mu$ sections were cut and stained with haematoxylin and eosin. The microscopical examination showed several small nodules in the submucosa, each of which consisted of a focus of fibrinoid necrosis surrounded by palisading histio- cytes. There was a moderate proliferation of endothelial cells and fibroblasts and an infiltration of plasma cells and lymphocytes in the fibrous connective tissue surrounding the nodules. The stratified squamous epithelium of the mucosa was normal. The nodules in the submucosal tissue of the vocal folds have the characteristic histology of rheumatoid nodules.

\section{Discussion}

The literature concerning rheumatoid involvement of the larynx places most emphasis on cricoarytenoid arthritis, and with good reason. Acute synovitis of these joints causes oedema and loss of mobility of the vocal folds with narrowing of the glottic chink, resulting in laryngeal obstruction. This may occur abruptly and be of such severity as to require emergency tracheostomy (Case 5 described by Polisar, 1959). In a similar case described by Pinals (1966), the acute cricoarytenoid arthritis was the presenting feature of rheumatoid arthritis. Chronic involvement with joint fibrosis leads to reduced mobility or even to fixation of one or both vocal folds in the midline position. The lesser degree of chronic laryngeal obstruction so produced may warrant arytenoidectomy for relief. Acute obstruction may also arise in this situation at any time (Vassalo, 1966): for instance, when oedema of the vocal folds develops during intercurrent hypopharyngeal infection or after endotracheal intubation for anaesthetic administration.

The autopsy findings reported by Wolman, Darke, and Young (1965) in eight rheumatoid arthritics with laryngeal involvement are worth further comment.

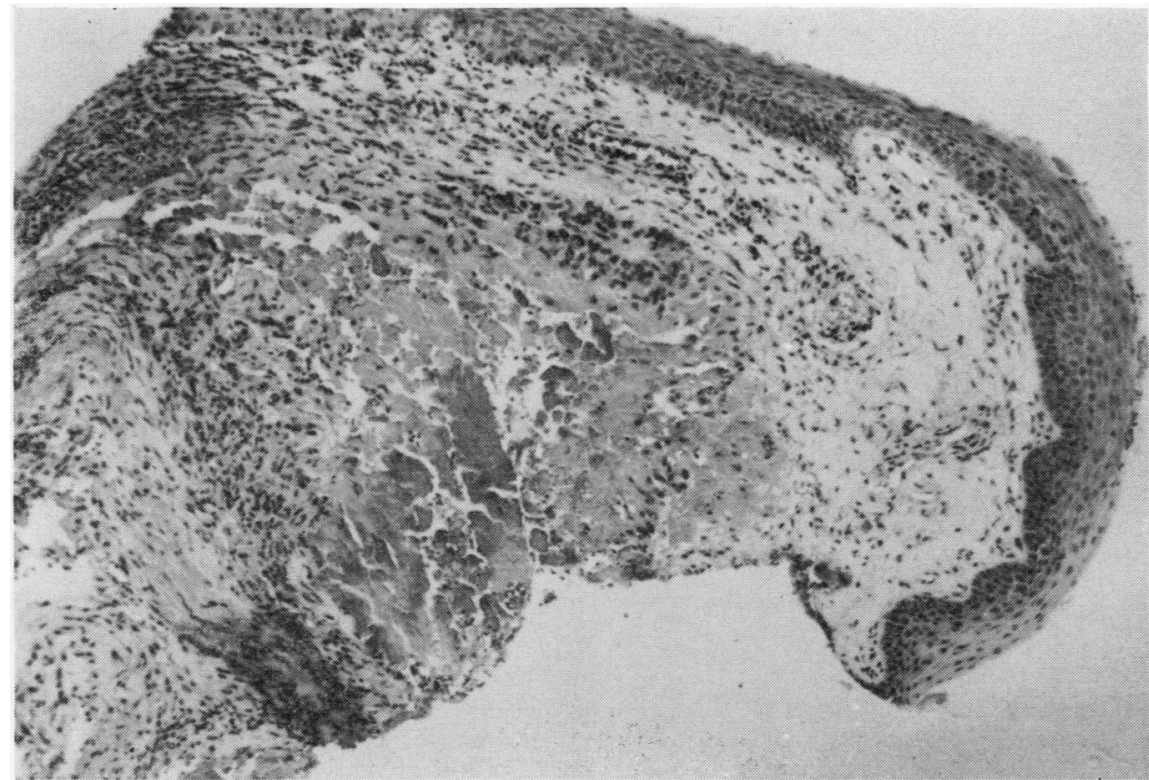

FIG. 3 A rheumatoid nodule in the submucosal fibrous tissue of the vocal fold. Haematoxylin and eosin. $\times 50$ 


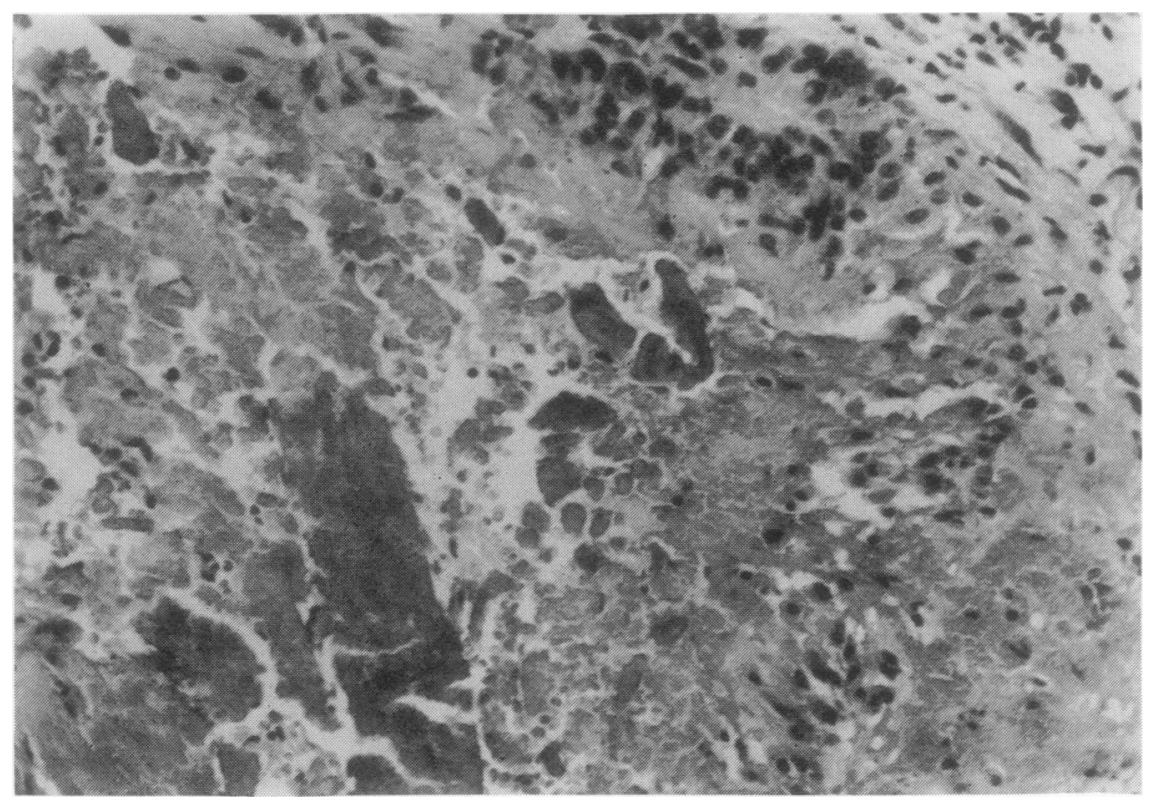

FIG. 4 Fibrinoid necrosis and palisading histiocytes of a rheumatoid nodule. Haematoxylin and eosin. $\times 128$

Besides involvement of the cricoarytenoid joints, they also found both active polymyositis and neural atrophy of the laryngeal musculature in some cases. The latter was secondary to ischaemic neuropathy of the recurrent laryngeal nerves caused by polyarteritis of both the vasa nervorum and the laryngeal branches of the thyroid arteries. In addition, necrotic mucosal ulcers of the hypopharynx, also due to arteritis, were present in one case.

Little significance appears to have been attached to the observed nodules and polyps described in the literature on rheumatoid laryngeal involvement. 'A few small submucous nodular thickenings in the region of the glottis, particularly one on the epiglottis' (the latter having the histological appearance of a necrobiotic granuloma) were reported by Raven, Parkes, Weber, and Prince (1948). Three of the cases reported by Polisar (1959) had glottic lesions: in Case 3 there was 'a polypoid pedunculated mass attached to the base of the epiglottis'; Case 4 showed 'a considerable amount of diffuse polypoid hypertrophy of the left vocal cord'; in Case 5 there was 'a polypoid pedunculated mass occupying the anterior half of the narrow glottic chink'. Histology of these lesions was not reported. Neither was histology obtained by Bienenstock, Ehrlich, and Freyberg (1963) for their patient M.S., described as having a $3 \times 5 \mathrm{~mm}$. polyp on each vocal fold. However, their patient J.C. was found at autopsy to have a histologically typical rheumatoid nodule in the muscles adjacent to the arytenoid cartilages. The previously mentioned patient of Pinals (1966) later developę̧ complete fixation of the vocal folds as well as 'as. nodular swelling at the posterior aspect of the right cord. Biopsy showed a chronic inflammatory reaction with infiltration of lymphocytes, histiocytes, and plasma cells'.

The case reported here firmly documents the occurrence of rheumatoid nodules involving the vocal folds. The symptoms in this case were due solely to their presence, as there was no clinical evidence of cricoarytenoid arthritis on a number of occasions. The glottic nodules and polyps previously observed in patients with rheumatoid cricoarytenoid arthritis, quoted above as originally described in the cited literature, probably represent further examples of rheumatoid vocal fold nodules. Histological examination of such lesions should always be performed to exclude more serious pathology.

\section{Summary}

The unusual occurrence of rheumatoid nodules of the vocal folds complicating a case of classical rheumatoid arthritis is reported, and the literature relevant to rheumatoid involvement of the larynx is briefly reviewed.

We wish to thank Dr. R. G. Robinson, Consultant Rheumatologist, and Dr. J. Dowe, Consultant Ear, Nose and Throat Surgeon, for their advice in the management of this patient and their interest in the preparation of this paper. 


\section{References}

Bienenstock, H., Ehrlich, G. E., AND Freyberg, R. H. (1963) Arthr. and Rheum., 6, 48 (Rheumatoid arthritis of the cricoarytenoid joint: a clinicopathologic study)

LOFGRen, R. H., AND MONTGOMERY, W. W. (1962) New Engl. J. Med., 267, 193 (Incidence of laryngeal involvement in rheumatoid arthritis)

PINALS, R. S. (1966) Brit. med. J., 1, 842 (Rheumatoid arthritis presenting with laryngeal obstruction)

PolisAR, I. A. (1959) Laryngoscope, 69, 1129 (The cricoarytenoid joint)

Raven, R. W., Weber, F. Parkes, AND Price, L. W. (1948) Ann. rheum. Dis., 7, 63 (The necrobiotic nodules of rheumatoid arthritis).

VASSALO, C. L. (1966) Arch. intern. Med., 117, 273 (Rheumatoid arthritis of the cricoarytenoid joints)

Wolman, L., DARKE, C. S., AND YounG, A. (1965) J. Laryng., 79, 403 (The larynx in rheumatoid arthritis) 